\title{
OPEN Development of high-resolution melting (HRM) assay to differentiate the species of Shigella isolates from stool and food samples
}

Babak Pakbin ${ }^{1,3}$, Afshin Akhondzadeh Basti ${ }^{3 凶}$, Ali Khanjari ${ }^{3}$, Wolfram Manuel Brück ${ }^{1}$ Leila Azimi ${ }^{2}$ \& Abdollah Karimi ${ }^{2}$

Shigella species, a group of intracellular foodborne pathogens, are the main causes of bacillary dysentery and shigellosis in humans worldwide. It is essential to determine the species of Shigella in outbreaks and food safety surveillance systems. The available immunological and molecular methods for identifying Shigella species are relatively complicated, expensive and time-consuming. High resolution melting (HRM) assay is a rapid, cost-effective, and easy to perform PCR-based method that has recently been used for the differentiation of bacterial species. In this study, we designed and developed a PCR-HRM assay targeting rrsA gene to distinguish four species of 49 Shigella isolates from clinical and food samples and evaluated the sensitivity and specificity of the assay. The assay demonstrated a good analytical sensitivity with $0.01-0.1 \mathrm{ng}$ of input DNA template and an analytical specificity of $100 \%$ to differentiate the Shigella species. The PCR-HRM assay also was able to identify the species of all 49 Shigella isolates from clinical and food samples correctly. Consequently, this rapid and user-friendly method demonstrated good sensitivity and specificity to differentiate species of the Shigella isolates from naturally contaminated samples and has the potential to be implemented in public health and food safety surveillance systems.

Shigella is the main cause of bacillary dysentery or shigellosis. It is an endemic infectious intestinal disease throughout the world and one of the major causes of mortality and morbidity, mostly among children up to 5 years old, especially in developing countries ${ }^{1}$. The main symptoms of bacillary dysentery include severe bloody diarrhoea accompanied by gastrointestinal cramps. However, the general symptoms of Shigella intestinal infections range from mild watery to acute diarrhoea depending on the type of the species which causes the infection ${ }^{2}$. Bacillary dysentery is caused by one of the species of Shigella consisting of S. dysenteriae, S. flexneri, S. Boydii, and S. sonnei. The infectious dose of Shigella species is very low from 10 (for S. dysenteriae) to 100 bacterial cells (for S. sonnei). Because of their low infectious dose, the control of outbreaks caused by Shigella species is difficult ${ }^{3}$. In addition, due to the release of Shiga-toxin, $S$. dysenteriae causes an acute extraintestinal disease in humans, hemorrhagic uremic syndrome (HUS) ${ }^{4}$. Annually, more than 165 million cases and 1.1 million related deaths caused by Shigella infections are recorded around the world especially in developing and low-income countries. $S$. sonnei and S. flexneri are the predominant species of Shigella in developed and developing countries, respectively ${ }^{5}$. Foods are the main serious route of Shigella species transmission to humans, causing related foodborne intestinal and extraintestinal diseases ${ }^{6}$. Reliable, rapid, and accurate differentiation between the species of Shigella is crucial to evaluate the suspected food and clinical samples in public health and food safety surveillance systems ${ }^{7}$.

Shigella strains are susceptible. While they are excreted in a sufficient number in food and stool, they die off rapidly due to environmental conditions, including $\mathrm{pH}$ and temperature. Therefore, classic microbiological methods used to identify and differentiate Shigella species isolated from food and stool samples are relatively

${ }^{1}$ Institute for Life Technologies, University of Applied Sciences Western Switzerland Valais-Wallis, 1950 Sion 2, Sierre, Switzerland. 'Pediatric Infections Research Center, Research Institute of Children's Health, Shahid Beheshti University of Medical Sciences, Tehran, Iran. ${ }^{3}$ Present address: Department of Food Hygiene and Quality of Control, Faculty of Veterinary Medicine, University of Tehran, P.O. Box: 14155-6453, Tehran, Iran. ${ }^{\square}$ email: aakhond@ut.ac.ir 


\begin{tabular}{|l|l|l|l|}
\hline \multirow{2}{*}{ Gene target } & Primer sequence $\left(\mathbf{5}^{\prime} \mathbf{3}^{\prime} \mathbf{}^{\prime}\right)$ & Amplicon size (bp) & Reference \\
\hline \multirow{2}{*}{ purA } & F-CTGGGTATCCTCAAAGCTTACTC & \multirow{2}{*}{83} & \multirow{2}{*}{ This study } \\
\cline { 2 - 4 } & R-AGGAACTCGCCAGTTTCATC & & \multirow{2}{*}{ This study } \\
\cline { 2 - 4 } & F-ATGCAAGTCGAACGGTAACA & \multirow{2}{*}{92} & \\
\cline { 2 - 4 } & R-CCCTCCATCAGGCAGTTTC & & \\
\hline
\end{tabular}

Table 1. Primer sequences used to identify and differentiate Shigella spp. by HRMA.

A.

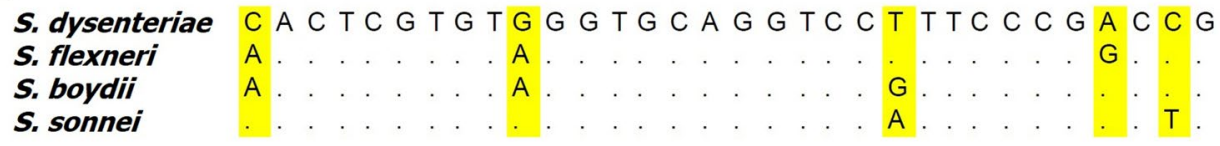

B.

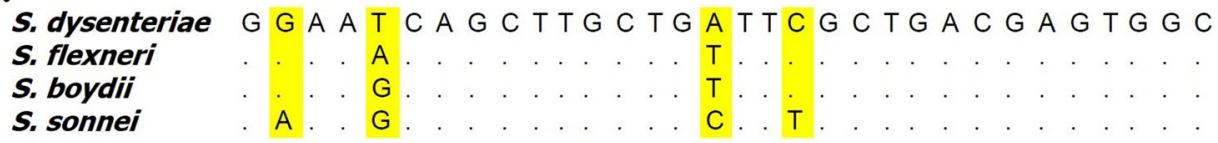

Figure 1. Nucleotide sequence alignments of S. dysenteriae (GenBank accession NC_007606), S. flexneri (NC_004741), S. boydii (NC_010658) and S. sonnei (NC_007384). (A) Primer pair purA-F-A and (B) primer pair rrsA-F-R.

time-consuming, expensive and inefficient ${ }^{8}$. Because of a low infectious dose, low numbers of causative bacteria in food and clinical samples, inappropriate sampling and competition from other commensal bacteria, the diagnosis of Shigella species still remains obscure ${ }^{9}$. To date, few methods have been designed and developed for the diagnosis of shigellosis and the identification of Shigella species in industrial and developing countries. The standard gold method to discriminate Shigella species are species-specific serologic assays ${ }^{10}$. Also, molecular methods have been developed to differentiate Shigella species such as conventional multiplex PCR $^{8}$, immunocapture $\mathrm{PCR}^{11}$, matrix-assisted laser desorption ionisation-time of flight mass spectrometry (MALDI-TOF MS) ${ }^{12}$, microarrays ${ }^{13}$, liquid chromatography-mass spectrometry (LC-MS) ${ }^{14}$ and next-generation sequencing (NGS) ${ }^{10}$ techniques. These assays have the advantage of being rapid. However, they are also expensive and complicated in their implementation. We conclude that a simple, rapid, inexpensive, accurate, specific, and sensitive method must be designed and developed for differentiation of Shigella species isolated from stool and food samples ${ }^{10,15,16}$.

High resolution melting (HRM) is an assay which, coupled with PCR, is regarded as a simple, low cost, and rapid method to detect single nucleotide polymorphism (SNP) ${ }^{17}$. Based on the dissociation behaviour of the amplicons, the HRM assay characterises and discriminates the PCR products in a single reaction tube without any additional instruments or protocols. The method is easy to perform, and the results can be obtained within approximately two hours ${ }^{18}$. Because the main inherent drawback of the HRM assay is its inability to identify the different species of a strain in a single reaction tube simultaneously, several researchers strongly recommended it to be developed and used in diagnostic systems ${ }^{19}$. PCR-HRM assay has already been used successfully to differentiate species of bacterial pathogens such as Staphylococcus ${ }^{20}$, Listeria $^{21}$, Cronobacter ${ }^{22}$, Salmonella ${ }^{23}$, Mycobacteria $^{24-26}$, Pasteurella $^{27}$, Campylobacter ${ }^{28}$, Yersinia ${ }^{29}$, and Brucella ${ }^{30}$ isolated from food and clinical samples. Thus far, no studies have focused on designing and developing PCR-HRM assays to distinguish Shigella species from each other. In this study, we developed a PCR-HRM method to differentiate four species of Shigella isolated from stool and food samples.

\section{Results}

Design of the primers. Using the specific sequences reported for adenylosuccinate synthetase and $16 \mathrm{~s}$ rRNA encoding genes of Shigella species, two pairs of primers, including purA-F-R and rrsA-F-R (Table 1), were designed and evaluated in this study. PurA-F-R and rrsA-F-R primers were designed to target the SNPs in adenylosuccinate synthetase and $16 \mathrm{~s}$ rRNA encoding genes, respectively unique to each species of Shigella to differentiate them from each other accurately. Primers purA-F-R and rrsA-F-R amplified 83 and 92 bp fragments, respectively (Table 1). The theoretical or in-silico melting temperatures calculated by uMelt Quartz online tool for S. dysenteriae, S. flexneri, S. Boydii, and S. sonnei with purA-F-R primers were 70.6, 70.4, 70.1 and $69.5^{\circ} \mathrm{C}$, respectively. While for rrsA-F-R primers melting temperatures were $71.6,71.9,72.6$ and $72.1{ }^{\circ} \mathrm{C}$ for $S$. dysenteriae, S. flexneri, S. boydii, and S. sonnei, respectively. As shown in Fig. 1, at least 2 different bases in the amplified sequences for both purA-F-R and rrsA-F-R primers in each Shigella species resulted in significant differences in the HRM profiles and melting temperatures. Both primers were also be able to amplify specific regions of Shigella species DNA by conventional PCR method. The purA-F-R primers were be able to generate the amplicons from 55 to $58.5^{\circ} \mathrm{C}$. However, rrsA-F-R primers amplicons were generated from 57.5 to $60{ }^{\circ} \mathrm{C}$. In this study, we 
A

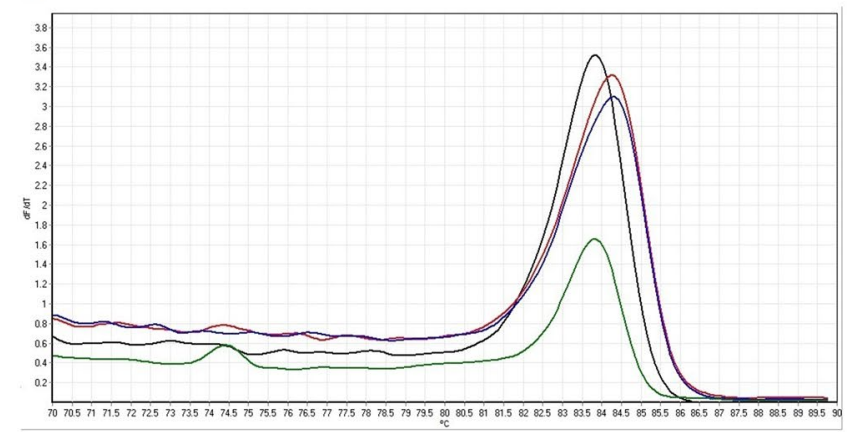

B

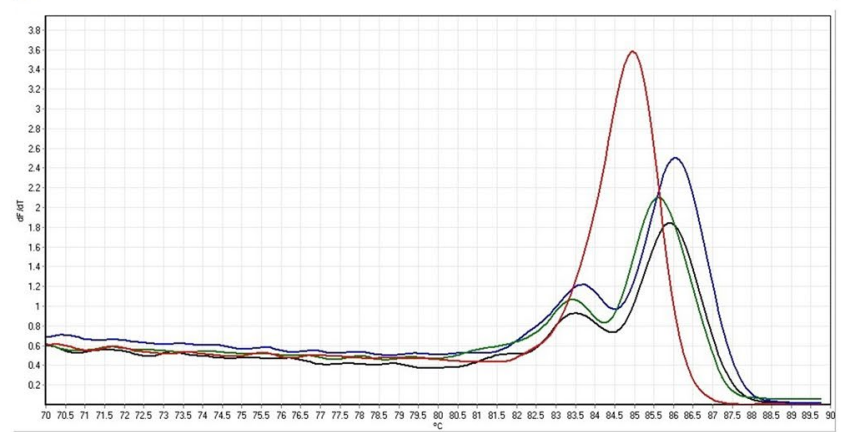

Figure 2. Melting curves of the reference strains of Shigella species analysed by PCR-HRM using (A) purA-F-R and (B) rrsA-F-R primer pairs. Green curve: S. dysenteriae. Red curve: S. flexneri. Blue curve: S. boydii. Black curve: S. sonnei.
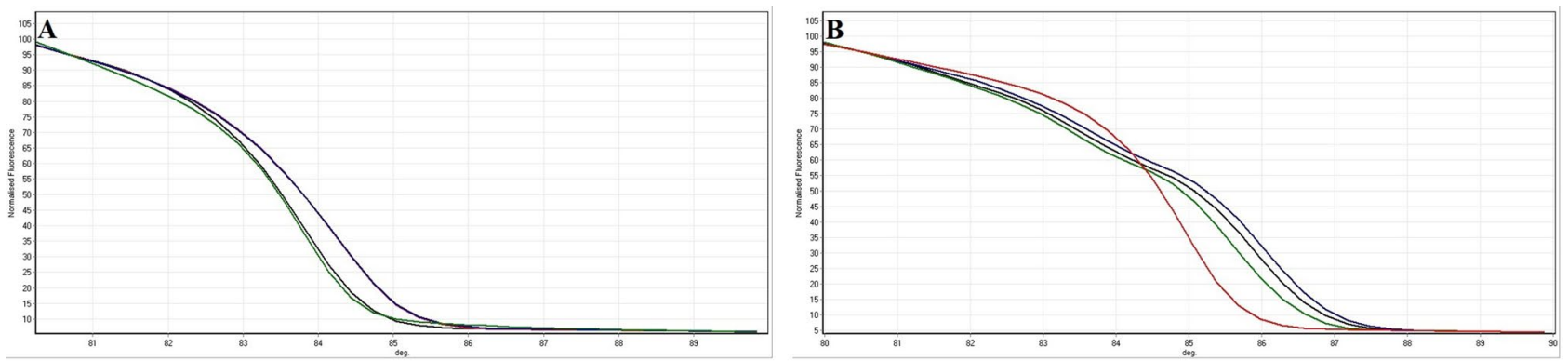

Figure 3. Normalised melting curves of the reference strains of Shigella species analysed by PCR-HRM using (A) purA-F-R and (B) rrsA-F-R primer pairs. Green curve: S. dysenteriae. Red curve: S. flexneri. Blue curve: $S$. boydii. Black curve: $S$. sonnei.
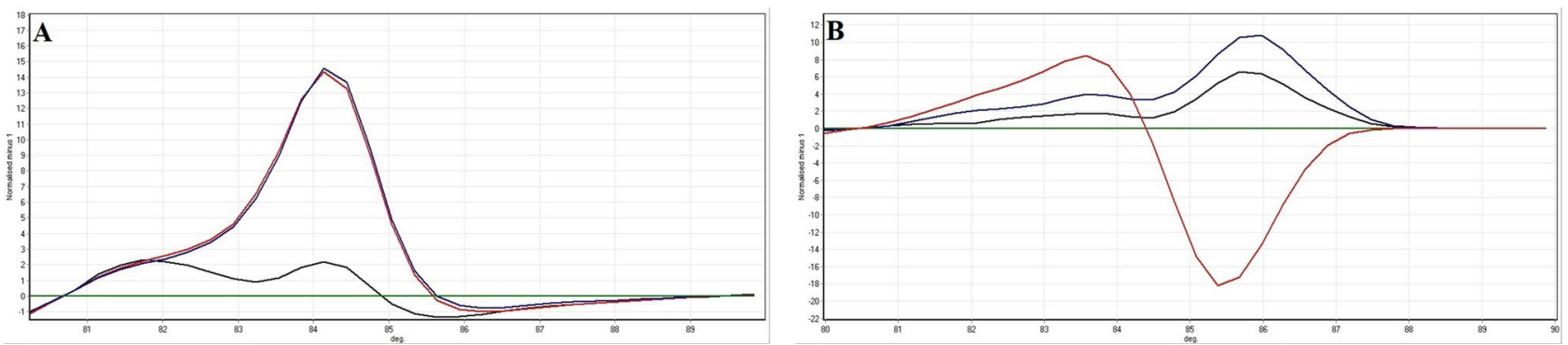

Figure 4. Difference plots of the reference strains of Shigella species analysed by PCR-HRM using (A) purAF-R and (B) rrsA-F-R primer pairs. Green curve: S. dysenteriae. Red curve: S. flexneri. Blue curve: S. boydii. Black curve: S. sonnei.

found that the optimal annealing temperatures for purA-F-R and rrsA-F-R primers in PCR-HRM assay were 58 and $59^{\circ} \mathrm{C}$, respectively. HRM investigated the effect of variations in these amplified sequences allowed us to differentiate Shigella species isolated from food and clinical samples.

HRM with the reference strains. Corresponding melting curves with definite melting temperatures were obtained from amplification of cultured reference strains tested in the PCR-HRM assay in this study. Figure 2A, B showed the HRM Shigella species-specific corresponding melting curves of the amplicons using purA-F-R and rrsA-F-R primers, respectively. Also, normalised curves and difference plots of the PCR-HRM assay using both primers (A: purA-F-R and B: rrsA-F-R) are demonstrated in Figs. 3 and 4, respectively. Melting temperatures of the amplicons generated by purA-F-R and rrsA-F-R primers to differentiate Shigella species by PCR-HRM method are presented in Table 2. As shown in Table 2, there were no significant $(P<0.01)$ differences between the melting temperature of $S$. dysenteriae and S. sonnei as well as and S. flexneri and S. boydii amplicons generated by purA-F-R primers demonstrating that these primers were not be able to differentiate Shigella species (confidence level $>90 \%)$. However, the PCR-HRM method with rrsA-F-R primers, designed and developed in this study, discriminated the four cultured species of Shigella from each other successfully and significantly $(P<0.01)$. Regard- 


\begin{tabular}{|l|l|l|}
\hline Shigella spp. & $\mathrm{T}_{\mathrm{m}} \pm \mathbf{S D}\left({ }^{\circ} \mathbf{C}\right)$-Primer purA & $\mathrm{T}_{\mathrm{m}} \pm \mathbf{S D}\left({ }^{\circ} \mathbf{C}\right)-$ Primer rrs $A$ \\
\hline S. dysenteriae & $83.75 \pm 0.05^{\mathrm{a}}$ & $85.61 \pm 0.04^{\mathrm{a}}$ \\
\hline S. flexneri & $84.28 \pm 0.06^{\mathrm{b}}$ & $84.69 \pm 0.05^{\mathrm{b}}$ \\
\hline S. Boydii & $84.24 \pm 0.05^{\mathrm{b}}$ & $86.03 \pm 0.05^{\mathrm{c}}$ \\
\hline S. sonnei & $83.85 \pm 0.06^{\mathrm{a}}$ & $85.90 \pm 0.06^{\mathrm{d}}$ \\
\hline
\end{tabular}

Table 2. Melting temperatures in PCR-HRM assay of the control strains of Shigella species. SD Standard deviation, $T_{m}$ Melting temperature. Different letters in each column showed significant differences $(P<0.01)$.
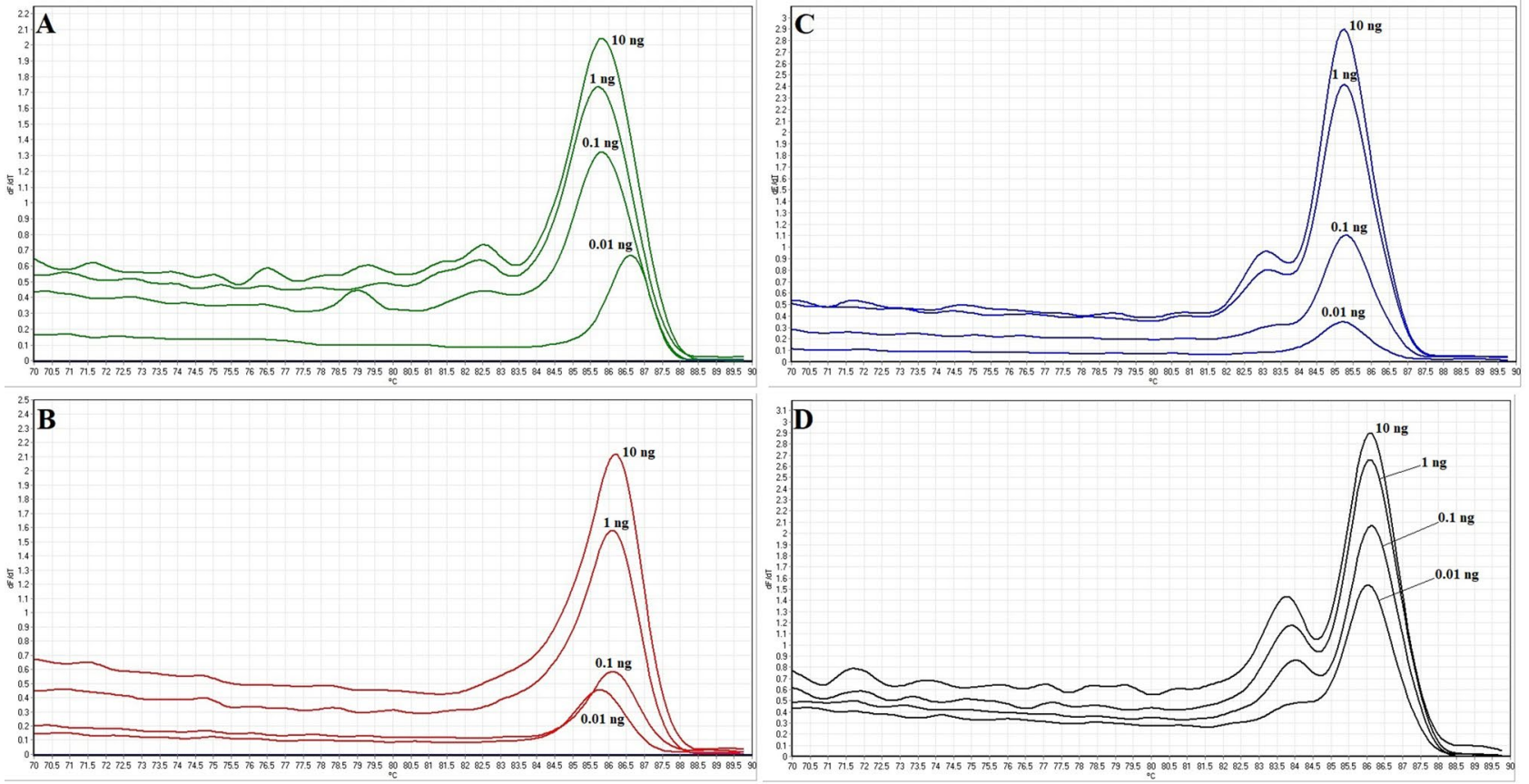

Figure 5. Melting curves of DNA tenfold dilution series for (A) S. dysenteriae, (B) S. flexneri, (C) S. boydii and (D) S. sonnei.

ing Figs. 2, 3 and 4, normalised curves and difference plots also demonstrated that four species of Shigella could be differentiated by the PCR-HRM assay using rrsA-F-R primers (confidence level $>90 \%$ ). However, purA-F-R primers were not able to distinguish Shigella species from each other correctly. Consequently, we were encouraged to use and evaluate PCR-HRM assay using rrsA-F-R primers, which have successfully been designed and developed at the present study and were be able to distinguish the Shigella species correctly for differentiation of Shigella species isolated from clinical and food samples (confidence level $>90 \%$ ).

Sensitivity and specificity of the PCR-HRM method. In this study, we found that the PCR-HRM assay using rrsA-F-R primers was able to differentiate the Shigella species correctly. PurA-F-R primers did not. As a result, the specificity of the PCR-HRM assay was calculated $100 \%$ for the identification and differentiation of all Shigella species. The sensitivity or limit of detection of the assay was measured by analysis of the serial dilutions of the DNA templates using the PCR-HRM assay (confidence level $>90 \%$ ). Melting and normalised melting curves of the serially diluted DNA templates analysed by PCR-HRM to identify four species of Shigella are shown in Figs. 5 and 6, respectively. The lowest concentrations of DNA by which the S. dysenteriae, S. flexneri, S. Boydii, and S. sonnei strains were identified correctly using the PCR-HRM assay, was $0.1,0.1,0.01$ and $0.01 \mathrm{ng}$, respectively (confidence level $>90 \%$ ). Consequently, the assay's sensitivity or limit of detection to identify S. boydii and $S$. sonnei was significantly $(P<0.01)$ more than that to identify S. dysenteriae and S. flexneri strains, which is indicated in Table 3. Identification of each species was compared with the reference strain in each reaction (confidence level >90\%).

HRM with the isolates from clinical and food samples. Out of 412 stool specimens from children up to 5 years old with acute diarrhoea and 470 food samples including raw milk, minced meat and vegetable salad samples, 28 and 21 Shigella species were isolated, respectively. All Shigella isolates $(\mathrm{N}=49)$ were detected using the conventional culture-based method and identified by serological tests as the gold standards. Shigella isolates from clinical (6 S. dyenteriae, 7 S. flexneri, 5 S. boydii and $10 \mathrm{~S}$. sonnei isolates) and food samples (5 S. dyenteriae, 5 S. flexneri, 4 S. boydii and 7 S. sonnei isolates) are presented in Table 4. Presumptive Shigella isolates from food 

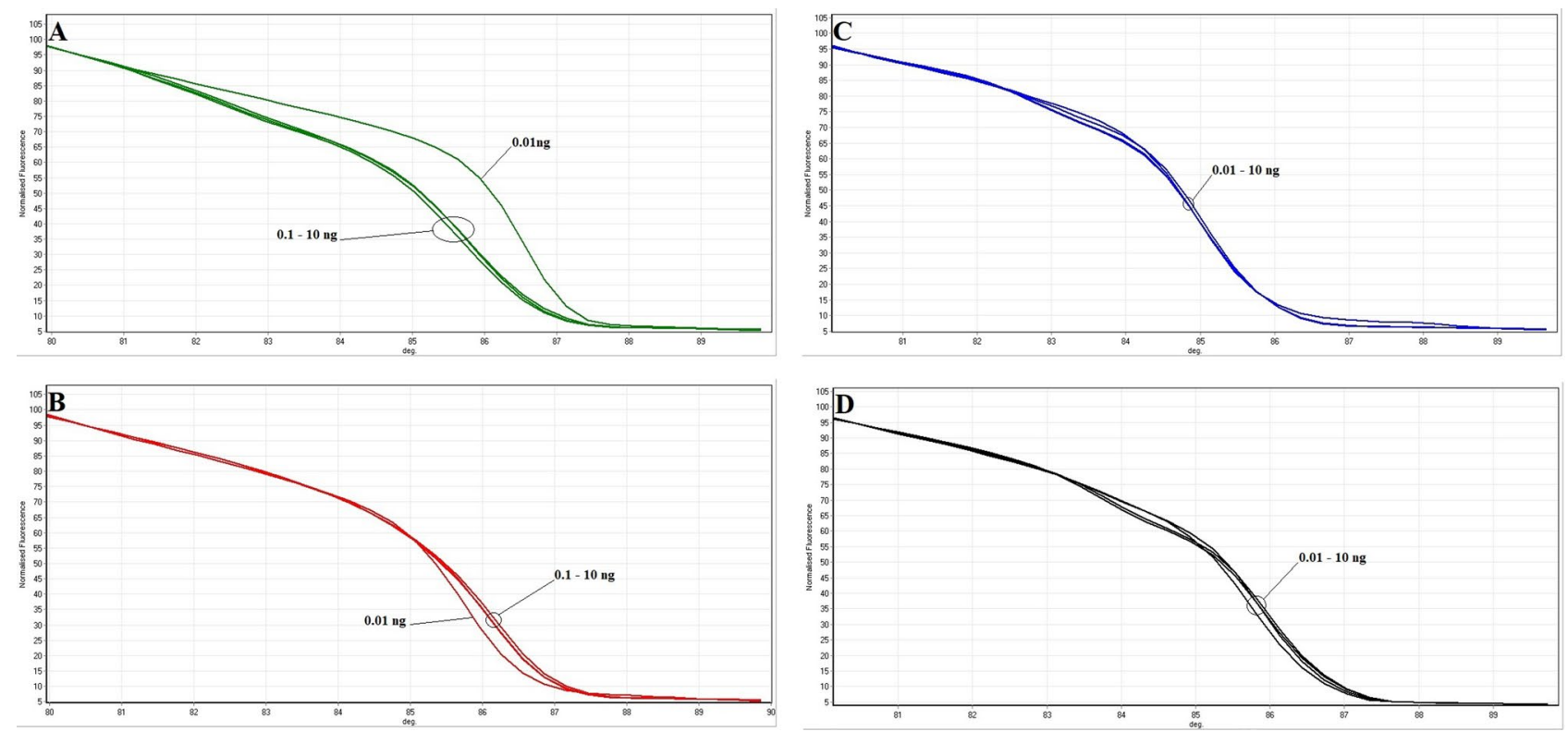

Figure 6. Normalised melting curves of DNA tenfold dilution series for (A) S. dysenteriae, (B) S. flexneri, (C) S. boydii and (D) S. sonnei.

\begin{tabular}{|c|c|c|c|c|}
\hline \multirow[b]{2}{*}{ Shigella spp. } & \multicolumn{4}{|c|}{ DNA concentrations } \\
\hline & $10 \mathrm{ng}$ & $1 \mathrm{ng}$ & $0.1 \mathrm{ng}$ & $0.01 \mathrm{ng}$ \\
\hline S. dysenteriae & $85.65 \pm 0.03^{\mathrm{a}}$ & $85.60 \pm 0.03^{\mathrm{a}}$ & $85.60 \pm 0.02^{\mathrm{a}}$ & $86.60 \pm 0.04^{\mathrm{b}}$ \\
\hline S. flexneri & $86.30 \pm 0.05^{\mathrm{a}}$ & $86.32 \pm 0.02^{\mathrm{a}}$ & $86.30 \pm 0.05^{\mathrm{a}}$ & $85.70 \pm 0.03^{\mathrm{b}}$ \\
\hline S. Boydii ${ }^{\mathrm{NS}}$ & $85.30 \pm 0.01$ & $85.32 \pm 0.03$ & $85.33 \pm 0.03$ & $85.30 \pm 0.02$ \\
\hline S. sonnei ${ }^{\mathrm{NS}}$ & $86.10 \pm 0.03$ & $86.10 \pm 0.01$ & $86.15 \pm 0.03$ & $86.05 \pm 0.03$ \\
\hline
\end{tabular}

Table 3. Melting temperatures $\left(\mathrm{T}_{\mathrm{m}} \pm \mathrm{SD}\left({ }^{\circ} \mathrm{C}\right)\right)$ in PCR-HRM assay of melting curves of DNA tenfold dilution series for all Shigella species using rrsA primer. SD Standard deviation, $T_{m}$ Melting temperature, NS Not significant, Different letters in each row showed significant differences $(P<0.01)$.

and clinical samples were subjected to DNA extraction and then PCR-HRM analysis using the rrsA-F-R primers designed and developed successfully in this study. High resolution melting graphs including melting curve, normalised melting curve and difference plots corresponding to the PCR-HRM of the Shigella isolates from clinical and food samples, are demonstrated in Figs. 7 and 8, respectively.

Regarding the melting curve, the normalised melting curve and difference plots, the PCR-HRM assay using rrsA-F-R primers was able tosignificantly categorise $(P<0.01)$ the amplicons into the four distinct main groups and showed a correct and successful Shigella species identification (confidence level $>90 \%$ ). Consequently, the PCR-HRM method developed in this study succeeded in detecting and differentiating all four species of Shigella isolates from clinical and food samples. These results also confirmed the high specificity and efficiency of the assay to differentiate Shigella species in naturally contaminated samples demonstrating the potential application of this method to the analysis of the isolates from clinical and food samples.

\section{Discussion}

Four species of Shigella including S. dysenteriae, S. flexneri, S. Boydii, and S. sonnei are among the main public health threats and food safety concerns around the world in developing and developed countries ${ }^{3,5}$. Nowadays, rapid and conventional methods such as hybridisation probes, multiplex PCR with species-specific primers, biochemical identification and serologic tests, which are so expensive or time consuming, have been developed and used to identify and differentiate four species of Shigella in food and clinical isolates ${ }^{10,14,16}$. Elahi et al. recently reported the development of a fluorescence DNA probe nano-biosensor method based on iron and gold nanoparticle to identify different species of Shigella ${ }^{31}$. However, this method is more expensive and complicated due to the need for four different probes bound to the gold and iron nanoparticles and the fluorescence spectrophotometer system to measure the fluorescence intensity and characterise possible interactions between the nanoparticles ${ }^{32}$. PCR-HRM is a rapid, simple, and cost-effective assay to detect SNPs in the PCR amplicons of the same size and has been highly recommended by several researchers to be developed and used for differentiation of pathogenic bacterial species isolated from clinical and food samples. It is worth noting that PCR-HRM assay can differentiate the species based on SNPs while the SNPs contribute to significant changes in the melting 


\begin{tabular}{|c|c|c|c|}
\hline Bacterial species & Strain designation & Source & Serogroup \\
\hline S. dysenteriae & MMRC2017241 & Stool & A \\
\hline S. dysenteriae & MMRC2017242 & Stool & $\mathrm{A}$ \\
\hline S. dysenteriae & MMRC2017243 & Stool & A \\
\hline S. dysenteriae & MMRC2017244 & Stool & A \\
\hline S. dysenteriae & MMRC2017245 & Stool & A \\
\hline S. dysenteriae & MMRC2017246 & Stool & A \\
\hline S. dysenteriae & PHSMS2019443 & Food & A \\
\hline S. dysenteriae & PHSMS2019444 & Food & A \\
\hline S. dysenteriae & PHSMS2019445 & Food & A \\
\hline S. dysenteriae & PHSMS2019446 & Food & A \\
\hline S. dysenteriae & PHSMS2019447 & Food & A \\
\hline S. flexneri & MMRC2017247 & Stool & B \\
\hline S. flexneri & MMRC2017248 & Stool & B \\
\hline S. flexneri & MMRC2017249 & Stool & B \\
\hline S. flexneri & MMRC2017250 & Stool & B \\
\hline S. flexneri & MMRC2017251 & Stool & B \\
\hline S. flexneri & MMRC2017252 & Stool & B \\
\hline S. flexneri & MMRC2017253 & Stool & B \\
\hline S. flexneri & PHSMS2019448 & Food & B \\
\hline S. flexneri & PHSMS2019449 & Food & B \\
\hline S. flexneri & PHSMS2019450 & Food & B \\
\hline S. flexneri & PHSMS2019451 & Food & B \\
\hline S. flexneri & PHSMS2019452 & Food & B \\
\hline S. boydii & MMRC2017254 & Stool & C \\
\hline S. boydii & MMRC2017255 & Stool & C \\
\hline S. boydii & MMRC2017256 & Stool & C \\
\hline S. boydii & MMRC2017257 & Stool & C \\
\hline S. boydii & MMRC2017258 & Stool & C \\
\hline S. boydii & PHSMS2019453 & Food & C \\
\hline S. boydii & PHSMS2019454 & Food & C \\
\hline S. boydii & PHSMS2019455 & Food & C \\
\hline S. boydii & PHSMS2019456 & Food & C \\
\hline S. sonnei & MMRC2017259 & Stool & $\mathrm{D}$ \\
\hline S. sonnei & MMRC2017260 & Stool & $\mathrm{D}$ \\
\hline S. sonnei & MMRC2017261 & Stool & $\mathrm{D}$ \\
\hline S. sonnei & MMRC2017262 & Stool & $\mathrm{D}$ \\
\hline S. sonnei & MMRC2017263 & Stool & $\mathrm{D}$ \\
\hline S. sonnei & MMRC2017264 & Stool & $\mathrm{D}$ \\
\hline S. sonnei & MMRC2017265 & Stool & $\mathrm{D}$ \\
\hline S. sonnei & MMRC2017266 & Stool & $\mathrm{D}$ \\
\hline S. sonnei & MMRC2017267 & Stool & $\mathrm{D}$ \\
\hline S. sonnei & MMRC2017268 & Stool & $\mathrm{D}$ \\
\hline S. sonnei & PHSMS2019457 & Food & $\mathrm{D}$ \\
\hline S. sonnei & PHSMS2019458 & Food & $\mathrm{D}$ \\
\hline S. sonnei & PHSMS2019459 & Food & $\mathrm{D}$ \\
\hline S. sonnei & PHSMS2019460 & Food & $\mathrm{D}$ \\
\hline S. sonnei & PHSMS2019461 & Food & $\mathrm{D}$ \\
\hline S. sonnei & PHSMS2019462 & Food & $\mathrm{D}$ \\
\hline S. sonnei & PHSMS2019463 & Food & $\mathrm{D}$ \\
\hline
\end{tabular}

Table 4. Shigella species isolated from clinical and food samples.

curves of the amplicons ${ }^{33,34}$. This study developed a PCR-HRM assay to identify and differentiate four species of Shigella and used it as an efficient method to differentiate all Shigella species isolated from reference cultured, naturally contaminated clinical and food samples. 

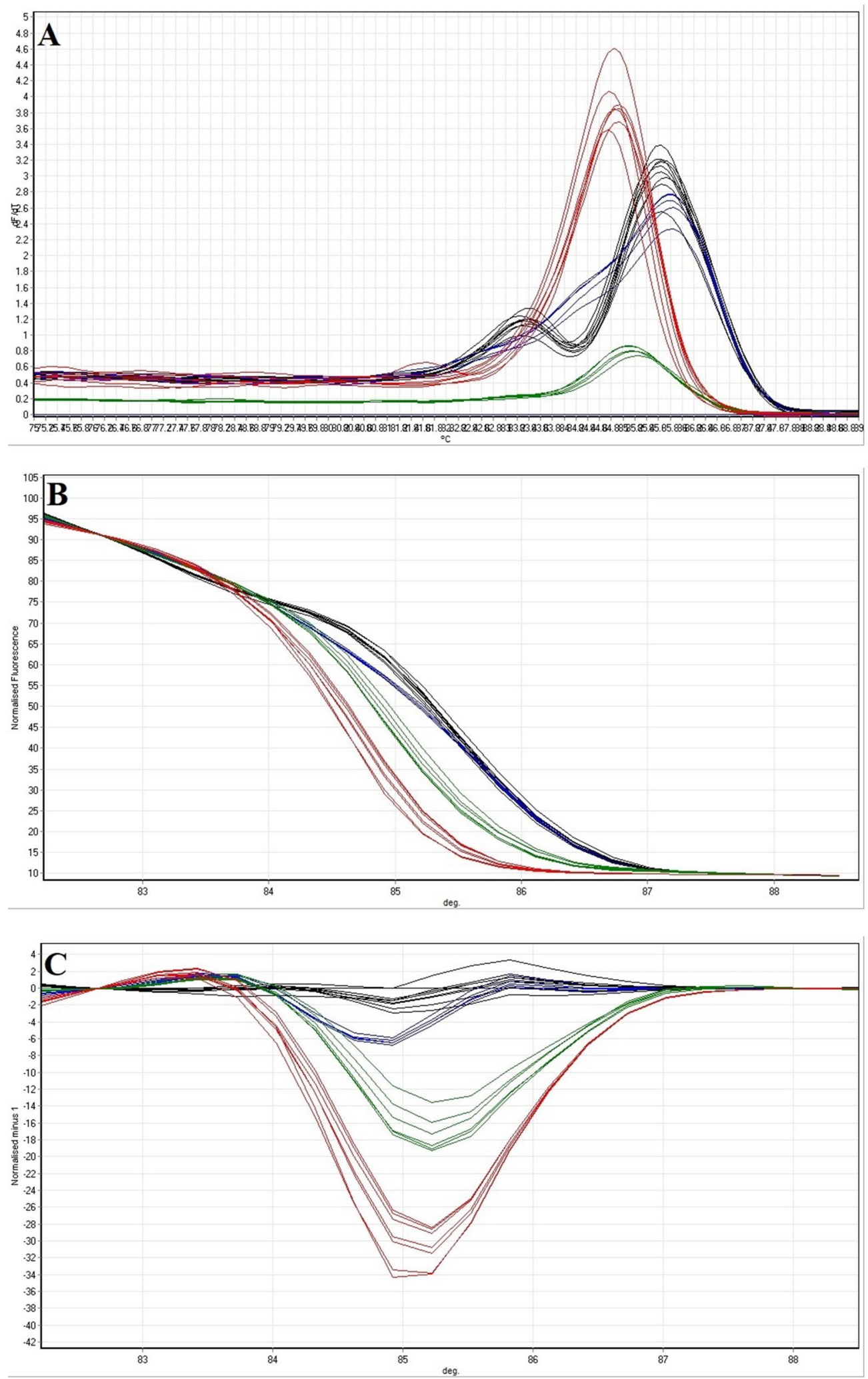

Figure 7. (A) Melting curves, (B) normalised melting curves and (C) difference plots the Shigella isolates from stool samples analysed by PCR-HRM assay. Green curve: $S$. dysenteriae. Red curve: S. flexneri. Blue curve: $S$. boydii. Black curve: S. sonnei. 

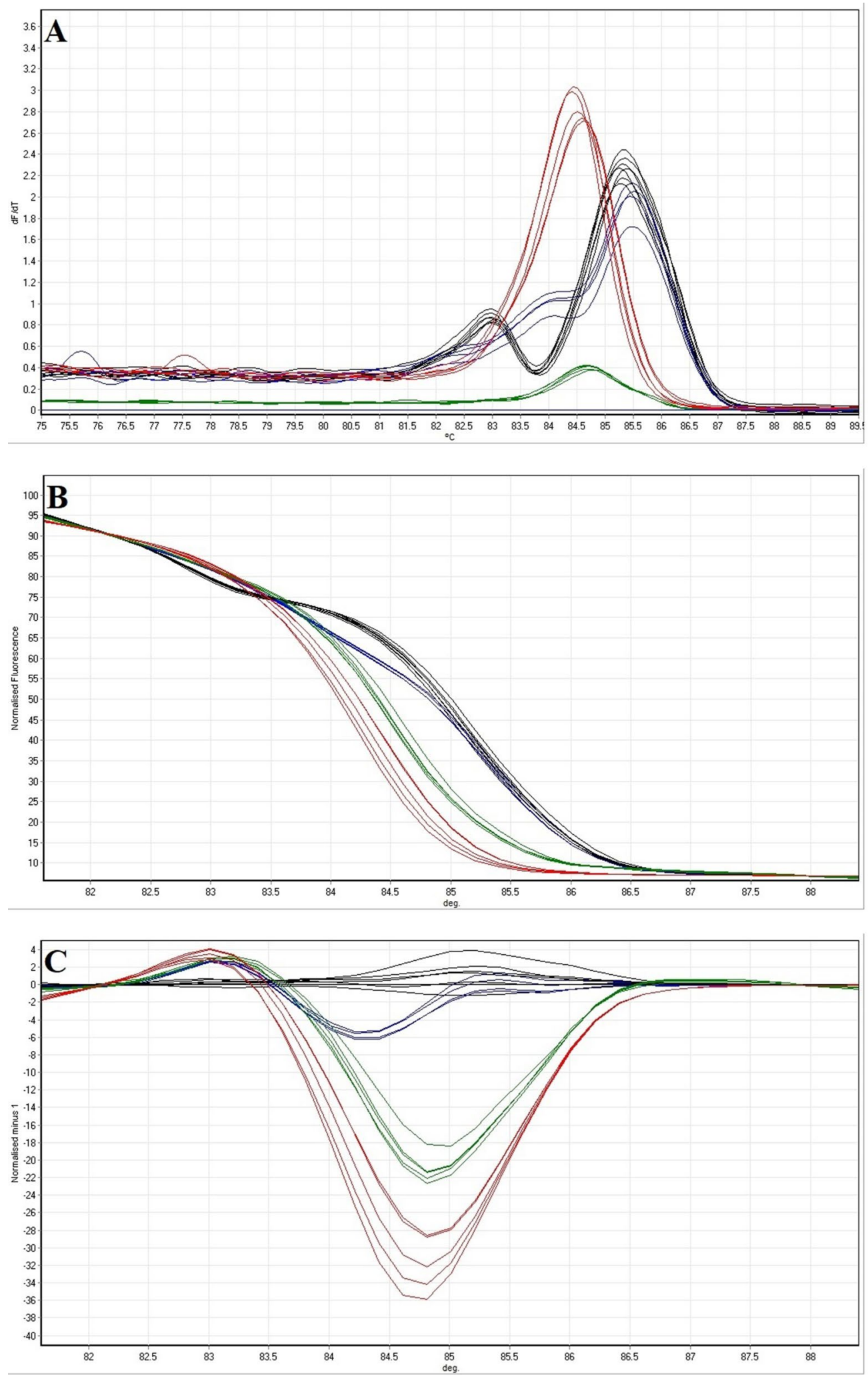

Figure 8. (A) Melting curves, (B) normalised melting curves and (C) difference plots the Shigella isolates from food samples analysed by PCR-HRM assay. Green curve: S. dysenteriae. Red curve: S. flexneri. Blue curve: $S$. boydii. Black curve: S. sonnei. 
We designed and developed two primer pairs, including purA-F-R and rrsA-F-R to amplify specific sequences of adenylosuccinate synthetase and $16 \mathrm{~s}$ rRNA encoding genes in Shigella species and distinguish the species from each other based on SNP differences among the amplified sequences by PCR-HRM assay. In order to develop an HRM assay and effective primer design, the amplified PCR products should not exceed than $150 \mathrm{bp}^{35}$. Both of these genes are conserved across the Shigella species and can be considered appropriate targets for PCR-HRM assay development to differentiate the species ${ }^{36}$. Ojha et al. developed a multiplex PCR assay and targeted $i n v C$, $r f c, w b g Z$ and $r f p B$ genes to discriminate the species of Shigella ${ }^{8}$. Radhika et al. also used $i p a H, w z y, w b g Z$ and invA genes to design and develop a multiplex PCR method for differentiation of Shigella species ${ }^{37}$. So far, no PCRHRM assay and the associated primers have been designed and developed to distinguish all species of Shigella. We demonstrated that Shigella species in cultured reference strains could be differentiated by the PCR-HRM assay using rrsA-F-R primers in a single round of reaction. However, the species were not discriminated from each other with purA-F-R primers. The DNA melting temperature and profile are strongly associated with the genetic variation and SNPs distribution patterns in the amplified sequences ${ }^{38}$. We demonstrated that nucleotide variations within the amplified sequences using rrsA-F-R primers were higher than that of purA-F-R primers. Also, higher distance differences were observed between the in-silico melting temperatures of the amplified sequences when the rrsA-F-R primers were used than purA-F-R primers.

Regarding the unique melting temperatures, normalised curves and difference plots, S. dysenteriae, S. flexneri, $S$. Boydii, and S. sonnei can be reliably and clearly distinguished from each other by the designed and developed PCR-HRM assay using the rrsA-F-R primers in this study. It is worthwhile to note that, considering the $\mathrm{T}_{\mathrm{m}}$ alone is not sufficient to differentiate the bacterial species ${ }^{39}$ and after a specific transformation of the melting curves into the normalised curve and difference plots by Rotor-Gene 6000 software, species of Shigella can be clearly discriminated into four distinct groups. Other studies also assessed the normalised curves and difference plots of the cultured reference strains ${ }^{40}$. The specificity of the assay was measured $100 \%$. The principal feature and advantage of our developed PCR-HRM method compared to the previous studies is the simple, straightforward and cheap PCR-based method to differentiate all Shigella species. Landolt et al. targeted the gyrB gene to develop a PCR-HRM assay for identification and differentiation of different species of Mycobacterium, including $M$. tuberculosis, M. microti, M. bovis, and M. capra. They successfully discriminated Mycobacterium species from each other with the analytical specificity of $100 \%$ by PCR-HRM assay and reported this method rapid, specific, low cost, and easy to perform in a single reaction tube ${ }^{24}$. Miller et al. used a PCR-HRM assay for identification and differentiation of six different reference species of Pasteurellaceae and exhibited six distinct melting profiles sufficiently distinguishable based on their $\mathrm{T}_{\mathrm{m}}$ values. They found PCR-HRM method-specific (100\%), rapid and cost-effective compared to the sequencing-based methods to identify and discriminate the Pasteurellaceae species ${ }^{27}$. To determine the sensitivity or LOD of the primers, a tenfold dilution series of the input DNA templates were prepared and analysed by the PCR-HRM assay ${ }^{23,40}$. Our developed assay demonstrated a good analytical sensitivity with a LOD of $0.01-0.1 \mathrm{ng}$ of the input DNA template to identify and differentiate the Shigella species when we used 30-cycle amplification PCR program. The melt temperature of the amplicons significantly shifted to a higher melting temperature when the concentration of the input DNA template decreased.

Consequently, the PCR-HRM assay is highly sensitive to the concentration of the DNA template ${ }^{41}$. Bender et al. evaluated PCR-HRM assay for identification of different pathogens, and they reported a suitable sensitivity with $0.5-1 \mathrm{ng}$ of input DNA template when performing 29-cycle PCR amplification. When the PCR cycles is increased, the sensitivity of the assay is consequently improved. However, this contributed to non-specific amplification $^{42}$.

It is essential to evaluate the molecular technique through analysis of the naturally contaminated samples ${ }^{10,25-28,42}$. We identified and differentiated the species of totally 49 Shigella isolates from clinical and food samples by the PCR-HRM assay using rrsA-F-R primers to evaluate this method for analysing samples collected from patients and foods. All 49 Shigella isolates were identified and distinguished correctly. We found this assay completely specific (100\%) and sensitive (100\%) to differentiate Shigella species isolated from stool and food samples showing concordant results with the previous studies. Landolt et al. evaluated the performance of PCRHRM assay to identify different species of Mycobacterium isolates from 25 clinical specimens, and they identified 23 isolates $(92 \%)$ correctly ${ }^{24}$. Slany et al. ${ }^{20}$, Souza et al. ${ }^{29}$, Hoseinpour et al. ${ }^{28}$, Wu et al..$^{43}$ and Cai et al. ${ }^{22}$ identified different species of Staphylococcus, Yersinia, Campylobacter, Helicobacter, and Cronobacter strains, respectively using PCR-HRM assay. All of these studies identified the microbial species isolated from naturally contaminated samples with a desirable specificity and sensitivity ${ }^{20,22,28,29,43}$. However, one of the main limitations of PCR-HRM assay generally is its inability to simultaneously identify more than one target or species in a test tube ${ }^{38}$. To the best of our knowledge, we developed the first Shigella species differentiation based on PCR-HRM assay and demonstrated this assay specific and sensitive to identify the species of Shigella isolates from real samples. This novel simple, rapid, cost effective and efficient molecular technique is highly recommended for designing and development of different diagnostic and identification tests. However, one of the most drawback of HRM assays is disability identify more than one species simultaneously while a single pair of primer is used. The design and development of multiple PCR-HRM assays to differentiate Shigella species are suggested to be implemented for future investigations.

\section{Conclusions}

We developed a new PCR-HRM assay to identify and differentiate four species of Shigella isolated from naturally contaminated clinical and food samples and presented the results. We designed and developed the PCR-HRM assay with the analytical specificity of $100 \%$ and good analytical sensitivity of $0.01-0.1 \mathrm{ng}$ of input DNA template concentration, indicating that this rapid method is sufficiently sensitive and specific for analysis of the isolates from real samples. Our assay also differentiated the species of all 49 Shigella isolates from clinical and food 
samples successfully. It is expected that a user friendly, rapid, cost-effective, sensitive, specific, and accurate PCRHRM assay could be developed and implemented in clinical and food microbiology laboratories to differentiate the species of Shigella infection and contaminations contributing to improving public health and food safety surveillance strategies around the world.

\section{Methods}

Samples and bacterial references. The clinical samples $(\mathrm{N}=412$, including stool specimens from children up to 5 years old with acute diarrhea referred to the pediatric emergency service and central lab of Qazvin children hospital, Qazvin, Iran) and the food samples $(\mathrm{N}=470$, including raw milk $(\mathrm{n}=130)$, minced meat $(n=160)$ and vegetable salad $(n=180)$ samples from different local markets located in different areas of municipality of Qazvin, Iran) were collected during July 2017 to February 2020. All samples were transported in cool boxes containing ice blocks $\left(4^{\circ} \mathrm{C} \pm 0.5\right)$ immediately to the central research laboratory, College of Veterinary Medicine, University of Tehran, Tehran, Iran, for further microbiological investigations. Four standard Shigella species including S. dysenteriae ATCC 13,313, S. flexneri PTCC 1865, S. boydii ATCC 12,030, and S. sonnei PTCC 1777 were used in this study as positive controls and reference strains. All bacterial cultures were purchased and obtained in lyophilised form from Pasteur Institute (Pasteur In., Tehran, Iran), inoculated in Trypticase Soy Broth (TSB, Merck, Germany) incubated at $37^{\circ} \mathrm{C}$ overnight before use. All bacterial strains were subjected to DNA extraction.

Detection and identification of Shigella species by culture-based methods in clinical and food samples. Shigella species were isolated and identified according to the methods described by Phiri et al. ${ }^{44}$ and Mokhtari et al. ${ }^{45}$. Using sterilised disposable inoculation loops, stool samples were directly plated and inoculated on xylose lysine deoxycholate (XLD) agar (Merck, Germany) and incubated for $24 \mathrm{~h}$ at $37^{\circ} \mathrm{C}$ aerobically. Suspected colonies, including red ones on XLD agar were selected, isolated and subjected to biochemical tests. We used the method previously described by Ahmed and Shimamoto ${ }^{46}$ to detect and identify Shigella species in different food samples. $10 \mathrm{~g}$ or $\mathrm{mL}$ of each sample (minced meat, raw milk and vegetable salad) was mixed vigorously with $100 \mathrm{~mL}$ Shigella broth (Merck, Germany) supplemented with Novobiocin antibiotic $\left(2 \mathrm{mg} \mathrm{L}^{-1}\right)$ (Merck, Germany), homogenised at $260 \mathrm{rpm}$ for $5 \mathrm{~min}$ and incubated anaerobically at $42{ }^{\circ} \mathrm{C}$ overnight. $100 \mu \mathrm{L}$ of the enriched samples were streaked onto XLD agar (Merck, Germany) plate and incubated aerobically at $37^{\circ} \mathrm{C}$ for $24 \mathrm{~h}$. Suspected colourless or red colonies on XLD agar isolated from stool and food samples were subjected to the biochemical tests, including IMViC, TSI, motility, oxidase and urease production. Also, presumptive Shigella isolates were identified and grouped serologically using commercial Shigella genus and species antisera kits (Difco Co., MI, USA) to confirm the genus and determine the species of the Shigella isolates, respectively.

DNA extraction. Presumptive Shigella isolates, colourless or red colonies on XLD agar, from food and clinical samples and the enriched reference bacterial strains were subjected to DNA extraction. Genomic DNA of the bacterial isolates and strains were extracted using the gram-negative bacterial DNA extraction kit (Sinaclon Co., Tehran, Iran) according to the manufacturer's protocol. The quality and quantity of the extracted genomes were evaluated using a NanoDrop 2000 spectrophotometer instrument (ThermoFisher, MD, USA). The final concentrations of all extracted genomes were adjusted to $50 \mathrm{ng} \mu \mathrm{L}^{-1}$ and all templates were kept at $-20{ }^{\circ} \mathrm{C}$ until further analysis.

Primer design. In this study, to differentiate Shigella species, the primers were designed based on targeting the highly conserved and variable regions of adenylosuccinate synthetase (pur) and $16 \mathrm{~s}$ rRNA (rrs) genes. The primers were designed according to the alignment of the available sequences of $\operatorname{pur} A$ and $\operatorname{rrs} A$ genes in Shigella species. We used GeneBank (NCBI, USA) sequence accession numbers NC_007606.1, NC_004741.1, NC_010658.1 and NC_007384.1 for S. dysenteriae, S. flexneri, S. boydii and S. sonnei, respectively. The sequences were aligned using CLUSTALW (EBI; http://www.ebi.ac.uk/CLUSTALW). PrimerQuest IDT online software (Integrated DNA Technologies, Inc, San Diego, CA, USA; https://www.idtdna.com/pages/tools/primerquest) was used to design two pairs of primers, purA-F-R and rrsA-F-R, to differentiate four species of Shigella. The quality of the designed primers was assessed by OligoAnalyzer online tool version 3.1 (https://eu.idtdna.com/ pages/tools/oligoanalyzer), and the primer specificity was evaluated using Primer-BLAST online tool (https:// www.ncbi.nlm.nih.gov/tools/primer-blast/). The primers were synthesised and purchased from CinnaGen company (Tehran, Iran).

Annealing temperature optimisation for primers. A temperature gradient program was designed and created in a thermocycler PCR machine (ABI, Applied Biosystems, CA, USA) to ensure that the primers designed and developed in this study were capable of amplification of the target regions in four Shigella species without any non-specific amplification and primer dimers demonstrating subsequent appropriate and desirable results in the following PCR-HRM analysis. Separate PCR reactions were performed for each pair of primers independently. PCR conditions included: $12.5 \mu \mathrm{L}$ of $2 \mathrm{X}$ PCR master mix (Ampliqon, Denmark), $1 \mu \mathrm{L}$ of each primer $\left(20 \mu \mathrm{mol} \mu \mathrm{L}^{-1}\right), 2 \mu \mathrm{L}$ of DNA template $\left(50 \mathrm{ng} \mu \mathrm{L}^{-1}\right)$ and sterilised DNase-free water up to the final reaction volume. Thermocycling conditions were the initial denaturation step including one cycle of $94{ }^{\circ} \mathrm{C}$ for 5 min; followed by 30 cycles of denaturation at $94{ }^{\circ} \mathrm{C}$ for $30 \mathrm{~s}$, annealing from 50 to $60{ }^{\circ} \mathrm{C}$ for $30 \mathrm{~s}$ and elongation at $72{ }^{\circ} \mathrm{C}$ for $20 \mathrm{~s}$; and a final extension step at $72{ }^{\circ} \mathrm{C}$ for $5 \mathrm{~min}$. The PCR products were characterised using gel electrophoresis on $2 \% \mathrm{w} / \mathrm{v}$ agarose/TBE buffer at $100 \mathrm{~V}$ for $60 \mathrm{~min}$ and visualised by UV transilluminator and gel documentation system (GelDoc model ccd-5, GenIranLab, Tehran, Iran). The primers were evaluated by using the reference strains and some Gram-positive and Gram-negative bacteria as the non-target strains. 
In-silico melting simulation. In-silico simulations of high-resolution melting curves were based on the target sequence regions amplified by purA-F-R (amplicon size: $83 \mathrm{bp}$ ) and rrsA-F-R (amplicon size: $92 \mathrm{bp}$ ) primers. The target sequences' theoretical melting temperatures $(\mathrm{Tm})$ were calculated using the uMelt Quartz online tool version 3.6.2 (https://www.dna-utah.org/umelt/quartz/um.php).

PCR-HRM. PCR-HRM was performed with a Solis-Bio 5X Evagreen ${ }^{\circledR}$ PCR-HRM hot start Master Mix (SolisBio Dyne, Tartu, Estonia) on a Rotor-Gene Q 6000 real-time PCR instrument (Corbett, Australia). PCR-HRM reaction tubes, performed for each primer pair separately, contained $4 \mu \mathrm{L}$ of the $5 \mathrm{X}$ PCR-HRM master mix, $1 \mu \mathrm{L}$ of each primer $(10 \mu \mathrm{M}), 2 \mu \mathrm{L}$ of the DNA template $\left(50 \mathrm{ng} \mu \mathrm{L}^{-1}\right)$ and DNase-free water to a final reaction volume of $20 \mu \mathrm{L}$. The amplification procedure was carried out using the following conditions: an initial denaturation step at $94{ }^{\circ} \mathrm{C}$ for $12 \mathrm{~min}$, followed by 30 cycles of denaturation at $94{ }^{\circ} \mathrm{C} 30 \mathrm{~s}$ and annealing-extension at 58 and $59^{\circ} \mathrm{C}$ for purA-f-R and rrsA-F-R, respectively for $30 \mathrm{~s}$; followed by HRM procedure with increasing temperature from 65 to $95{ }^{\circ} \mathrm{C}$ with data acquisition every $0.2{ }^{\circ} \mathrm{C}$ for both primer pairs. The sizes of amplicons produced by purA and rrsA primers were 83 and $92 \mathrm{bp}$, respectively (Table 1). The analysis of HRM was conducted using the Rotor-Gene 6000 software version 2.02 (Corbett, Australia) to determine the $T_{m}$, melting and normalised melting curves for each species of Shigella. For each experiment, S. dysenteriae ATCC 13,313, S. flexneri PTCC 1865, S. boydii ATCC 12,030, and S. sonnei PTCC 1777 were included as the positive controls and melting curve standards.

Sensitivity and specificity of the PCR-HRM method. To determine the sensitivity of the PCR-HRM method designed and developed in this study, serial tenfold dilutions $(10,1,0.1$ and $0.01 \mathrm{ng} \mu \mathrm{L})$ of the DNA templates of each reference Shigella species separately were prepared and analysed by the PCR-HRM assay. The sensitivity of the assay was measured as the lowest concentration with successful PCR amplification at which the Shigella species can be identified correctly by the developed PCR-HRM method. To check the possible incorrect signals and the specificity of the assay, differentiation and identification of four species of Shigella were performed among the Shigella species isolated from clinical and food samples by both the conventional serologybased (the gold standard) and the developed PCR-HRM methods.

Statistical analysis. One-way analysis of variance (ANOVA) was conducted to determine the significant $(P<0.01)$ differences among the groups of melting temperatures using SPSS version 23.0.0 (SPSS Inc., Chicago, IL, USA). Also, all experiments and measurements were performed in triplicates.

Ethics approval. The sampling and study protocols were investigated and approved by the Ethics Committee of the College of Veterinary Medicine, University of Tehran (IR.UT.REC.1397.204). In this study, all research was performed in accordance with relevant guidelines/regulations and the Declaration of Helsinki. For all cases, informed consent was obtained from the parents of the patients whose stool specimen was included in this study.

\section{Data availability}

All raw data in this study are available from the corresponding author on a reasonable request.

Received: 7 September 2021; Accepted: 23 December 2021

Published online: 10 January 2022

\section{References}

1. Baker, K. S. et al. Genomic epidemiology of Shigella in the United Kingdom shows transmission of pathogen sublineages and determinants of antimicrobial resistance. Sci. Rep. 8, 1-8 (2018).

2. Lima, I. F., Havt, A. \& Lima, A. A. Update on molecular epidemiology of Shigella infection. Curr. Opin. Gastroenterol. 31, 30-37 (2015).

3. Goldberg, M., Calderwood, S. B., Edwards, M. S. \& Bloom, A. Shigella infection: Epidemiology, microbiology, and pathogenesis. Recuperado el 14, 4-130 (2013).

4. Pakbin, B., Akhondzadeh Basti, A., Khanjari, A., Azimi, L. \& Karimi, A. Differentiation of stx1 A gene for detection of Escherichia coli serotype O157: H7 and Shigella dysenteriae type 1 in food samples using high resolution melting curve analysis. Food Sci. Nutr. 8, 3665-3672 (2020).

5. Kotloff, K. L. Shigella infection in children and adults: A formidable foe. Lancet Glob. Health 5, e1166-e1167 (2017).

6. Lampel, K. A. Shigella. pathogens and toxins in foods challenges and interventions 131-145 (ASM Press, 2009).

7. Pakbin, B. et al. Antibiotic susceptibility and genetic relatedness of Shigella species isolated from food and human stool samples in Qazvin Iran. BMC Research Notes 14, 1-6 (2021).

8. Ojha, S. C., Yean Yean, C., Ismail, A. \& Banga Singh, K.-K. A pentaplex PCR assay for the detection and differentiation of Shigella species. BioMed Res. Int. 2013, 1 (2013).

9. Ragupathi, N. D., Sethuvel, D. M., Inbanathan, F. \& Veeraraghavan, B. Accurate differentiation of Escherichia coli and Shigella serogroups: challenges and strategies. New microbes and new infections 21, 58-62 (2018).

10. Chattaway, M. A., Schaefer, U., Tewolde, R., Dallman, T. J. \& Jenkins, C. Identification of Escherichia coli and Shigella species from whole-genome sequences. J. Clin. Microbiol. 55, 616-623 (2017).

11. Peng, X., Luo, W., Zhang, J., Wang, S. \& Lin, S. Rapid detection of Shigella species in environmental sewage by an immunocapture PCR with universal primers. Appl. Environ. Microbiol. 68, 2580-2583 (2002).

12. Paauw, A. et al. Rapid and reliable discrimination between Shigella species and Escherichia coli using MALDI-TOF mass spectrometry. Int. J. Med. Microbiol. 305, 446-452 (2015).

13. Ndungo, E. et al. A novel Shigella proteome microarray discriminates targets of human antibody reactivity following oral vaccination and experimental challenge. Msphere 3, e00260-e218 (2018).

14. Lasch, P., Schneider, A., Blumenscheit, C. \& Doellinger, J. Identification of microorganisms by liquid chromatography-mass spectrometry (LC-MS1) and in silico peptide mass libraries. Mol. Cell. Proteom. 19, 2125-2139 (2020). 
15. Kim, H.-J., Ryu, J.-O., Song, J.-Y. \& Kim, H.-Y. Multiplex polymerase chain reaction for identification of Shigellae and four Shigella species using novel genetic markers screened by comparative genomics. Foodborne Pathog. Dis. 14, 400-406 (2017).

16. Bazhenova, A., Gao, F., Bolgiano, B. \& Harding, S. E. Glycoconjugate vaccines against Salmonella enterica serovars and Shigella species: existing and emerging methods for their analysis. Biophys. Rev. https://doi.org/10.1007/s12551-021-00791-z (2021).

17. Erali, M. \& Wittwer, C. T. High resolution melting analysis for gene scanning. Methods 50, 250-261 (2010).

18. Tong, S. Y. \& Giffard, P. M. Microbiological applications of high-resolution melting analysis. J. Clin. Microbiol. 50, 3418-3421 (2012).

19. Wittwer, C. T. High-resolution DNA melting analysis: Advancements and limitations. Hum. Mutat. 30, 857-859 (2009).

20. Slany, M. et al. Differentiation of Staphylococcus spp. by high-resolution melting analysis. Canadian J. Microb. 56, 1040-1049 (2010).

21. Ohshima, C. et al. Establishment of a simple and rapid identification method for Listeria spp. by using high-resolution melting analysis, and its application in food industry. PLoS One 9, e99223 (2014).

22. Cai, X.-Q. et al. Rapid detection and simultaneous genotyping of Cronobacter spp. (formerly Enterobacter sakazakii) in powdered infant formula using real-time PCR and high resolution melting (HRM) analysis. PLoS One 8, e67082 (2013).

23. Ren, X. et al. High resolution melting (HRM) analysis as a new tool for rapid identification of Salmonella enterica serovar Gallinarum biovars Pullorum and Gallinarum. Poult. Sci. 96, 1088-1093 (2017).

24. Landolt, P., Stephan, R. \& Scherrer, S. Development of a new High Resolution Melting (HRM) assay for identification and differentiation of Mycobacterium tuberculosis complex samples. Sci. Rep. 9, 1-8 (2019).

25. Issa, R. et al. High resolution melting analysis for the differentiation of Mycobacterium species. J. Med. Microbiol. 63, 1284-1287 (2014).

26. Khosravi, A. D., Hashemzadeh, M., Hashemi Shahraki, A. \& Teimoori, A. Differential identification of mycobacterial species using high-resolution melting analysis. Front. Microbiol. 8, 2045 (2017).

27. Miller, M., Zorn, J. \& Brielmeier, M. High-resolution melting curve analysis for identification of Pasteurellaceae species in experimental animal facilities. PLoS One 10, e0142560 (2015).

28. Hoseinpour, F., Foroughi, A., Nomanpour, B. \& Nasab, R. S. Identification and differentiation of Campylobacter species by highresolution melting curve analysis. Microb. Pathog. 108, 109-113 (2017).

29. Souza, R. A., Frazão, M. R., Almeida, A. M. \& Falcão, J. P. Rapid and efficient differentiation of Yersinia species using high-resolution melting analysis. J. Microbiol. Methods 115, 6-12 (2015).

30. Gopaul, K. K. et al. Development and assessment of multiplex high resolution melting assay as a tool for rapid single-tube identification of five Brucella species. BMC. Res. Notes 7, 1-12 (2014).

31. Elahi, N., Kamali, M., Baghersad, M. H. \& Amini, B. A fluorescence Nano-biosensors immobilisation on Iron (MNPs) and gold (AuNPs) nanoparticles for detection of Shigella spp. Mater. Sci. Eng. C 105, 110113 (2019).

32. Song, M., Yang, M. \& Hao, J. Pathogenic virus detection by optical nanobiosensors. Cell Rep. Phys. Sci. 2, 100288 (2021).

33. Druml, B. \& Cichna-Markl, M. High resolution melting (HRM) analysis of DNA-Its role and potential in food analysis. Food Chem. 158, 245-254 (2014).

34. Singh, P., Cubillos, G., Kirshteyn, G. \& Bosilevac, J. M. High-resolution melting real-time PCR assays for detection of Escherichia coli $\mathrm{O} 26$ and O111 strains possessing Shiga toxin genes. LWT 131, 109785 (2020).

35. Słomka, M., Sobalska-Kwapis, M., Wachulec, M., Bartosz, G. \& Strapagiel, D. High resolution melting (HRM) for high-throughput genotyping: Limitations and caveats in practical case studies. Int. J. Mol. Sci. 18, 2316 (2017).

36. Hendriks, A. C. et al. Genome-wide association studies of Shigella spp. and Enteroinvasive Escherichia coli isolates demonstrate an absence of genetic markers for prediction of disease severity. BMC Genom. 21, 1-12 (2020).

37. Radhika, M., Saugata, M., Murali, H. \& Batra, H. A novel multiplex PCR for the simultaneous detection of Salmonella enterica and Shigella species. Braz. J. Microbiol. 45, 667-676 (2014).

38. Farrar, J. S. \& Wittwer, C. Molecular diagnostics 79-102 (Elsevier, 2017).

39. Bosmali, I. et al. Novel authentication approach for coffee beans and the brewed beverage using a nuclear-based species-specific marker coupled with high resolution melting analysis. Lwt 137, 110336 (2021).

40. $\mathrm{Xu}$, S. et al. Direct detection of Corynebacterium striatum, Corynebacterium propinquum, and Corynebacterium simulans in sputum samples by high-resolution melt curve analysis. BMC Infect. Dis. 21, 1-9 (2021).

41. Keatley, S. et al. Species-level identification of trypanosomes infecting Australian wildlife by High-Resolution Melting-Real Time quantitative polymerase chain reaction (HRM-qPCR). Int. J. Parasitol. Parasites Wildlife 13, 261-268 (2020).

42. Bender, A. C., Faulkner, J. A., Tulimieri, K., Boise, T. H. \& Elkins, K. M. High Resolution Melt Assays to Detect and Identify Vibrio parahaemolyticus, Bacillus cereus, Escherichia coli, and Clostridioides difficile Bacteria. Microorganisms 8, 561 (2020).

43. Wu, M. et al. Differentiation of five enterohepatic Helicobacter species by nested PCR with high-resolution melting curve analysis. Helicobacter 22, e12362 (2017).

44. Phiri, A. F. et al. Burden, antibiotic resistance, and clonality of Shigella spp. implicated in community-acquired acute diarrhoea in Lilongwe Malawi. Tropical Med. Infect. Dis. 6, 63 (2021).

45. Mokhtari, W., Nsaibia, S., Gharbi, A. \& Aouni, M. Real-time PCR using SYBR Green for the detection of Shigella spp. in food and stool samples. Mol. Cellular Probes 27, 53-59 (2013).

46. Ahmed, A. M. \& Shimamoto, T. Molecular characterisation of multidrug-resistant Shigella spp. of food origin. Int. J. Food Microb. 194, 78-82 (2015).

\section{Acknowledgements}

The authors would like to thank Prof. Dr. John W.A. Rossen, Adjunct Professor, Department of Medical Microbiology and Infection Prevention University of Groningen, Groningen, The Netherlands for his editing of this manuscript.

\section{Author contributions}

B.P., A.A.B. and A.K. designed the research; B.P., A.K.H. and L.A. performed all experiments and analysis; B.P. and W.M.B. wrote the first draft of the manuscript; B.P., and W.M.B. finalised and reviewed the manuscript; all authors reviewed the draft and approved the manuscript.

\section{Competing interests}

The authors declare no competing interests.

\section{Additional information}

Correspondence and requests for materials should be addressed to A.A.B.

Reprints and permissions information is available at www.nature.com/reprints. 
Publisher's note Springer Nature remains neutral with regard to jurisdictional claims in published maps and institutional affiliations.

(c) (i) Open Access This article is licensed under a Creative Commons Attribution 4.0 International License, which permits use, sharing, adaptation, distribution and reproduction in any medium or format, as long as you give appropriate credit to the original author(s) and the source, provide a link to the Creative Commons licence, and indicate if changes were made. The images or other third party material in this article are included in the article's Creative Commons licence, unless indicated otherwise in a credit line to the material. If material is not included in the article's Creative Commons licence and your intended use is not permitted by statutory regulation or exceeds the permitted use, you will need to obtain permission directly from the copyright holder. To view a copy of this licence, visit http://creativecommons.org/licenses/by/4.0/.

(c) The Author(s) 2022 